IOS Press

\title{
Explanatory multivariate modeling for disability, pain, and claims in patients with spine pain via a physical therapy direct access model of care
}

Christopher E. Green ${ }^{\mathrm{a}}$, Anthony Pastore ${ }^{\mathrm{a}}$, Leah Cronley ${ }^{\mathrm{b}}$, Merritt D. Walker ${ }^{\mathrm{b}}$, Charles A. Thigpen ${ }^{\mathrm{c}, \mathrm{d}}$, Chad E. Cook ${ }^{\mathrm{b}}$ and Deborah L. Givens ${ }^{\mathrm{a}, *}$

${ }^{a}$ Division of Physical Therapy, Department of Allied Health Sciences, University of North Carolina at Chapel Hill, Chapel Hill, NC 27599, USA

${ }^{\mathrm{b}}$ Doctor of Physical Therapy Division, Department of Orthopaedics, Duke University, Durham, NC 27705, USA cATI Physical Therapy, University of South Carolina, Greenville, SC 29681, USA

${ }^{\mathrm{d}}$ Center for Effectiveness Research in Orthopaedics, University of South Carolina, Greenville, SC 29681, USA

\section{Abstract.}

BACKGROUND: Direct access physical therapy (DAPT) may result in improved patient outcomes and reduced healthcare costs. Prognostic factors associated with spine-related outcomes and insurance claims with DAPT are needed.

OBJECTIVE: To identify factors that predict variations in outcomes for spine pain and insurance claims using DAPT.

METHODS: Individuals $(\mathrm{N}=250)$ with spine pain were analyzed. Outcomes were classified into High, Low, or Did Not Meet minimal clinically important difference (MCID) scores. Claims were categorized into low, medium, or high tertiles. Prognostic variables were identified from patient information.

RESULTS: Females were more likely to meet High MCID (odds ratio [OR] 2.84 (95\% CI = 1.32, 6.11) and Low MCID (OR $2.86,95 \% \mathrm{CI}=1.34,6.10)$. Higher initial ODI/NDI scores were associated with High MCID $(\mathrm{OR} 1.04,95 \% \mathrm{CI}=1.07,1.22)$ and Low MCID (OR 0.91, 95\% CI $=0.77,1.07)$. Odds of a high claim were lowered by the absence of imaging $($ OR $0.04,95 \%$ $\mathrm{CI}=0.02,0.09)$ and an active versus passive treatment (OR $0.38,95 \% \mathrm{CI}=0.18,0.80)$.

CONCLUSION: Females and higher initial disability predicted favorable outcomes. The novel introduction of claims into the prognostic modeling supports that active interventions and avoiding imaging may reduce claims.

Keywords: Prognosis, low back pain, neck pain, health care costs, direct access

\section{Introduction}

Neck pain (NP) and low back pain (LBP) are two of the most common musculoskeletal conditions, with a global point prevalence of $4.9 \%$ [1] and $9.4 \%$ [2], re-

${ }^{*}$ Corresponding author: Deborah L. Givens, Division of Physical Therapy, UNC at Chapel Hill, 3032 Bondurant Hall, CB 7135, Chapel Hill, NC 27599, USA. Tel.: +1 919843 8660; E-mail: deborah_givens@med.unc.edu. spectively. In the United States (US), $15 \%$ of all individuals report experiencing NP and 29\% report LBP within the previous three months [3]. Whereas a majority of persons with NP and LBP may have favorable outcomes with or without treatment, a notable percentage of the population will go on to develop chronic or recurring pain and disability. Over a third of individuals who develop NP will develop persistent symptoms that last longer than six months [4]. Similarly, 24\% to $33 \%$ of individuals who experience activity-limiting 


\begin{abstract}
LBP will continue to have recurring episodes throughout their lifetime [5,6].

Numerous studies have examined prognostic factors related to NP and LBP and predicting disability based on these factors. There is strong evidence that older age, a longer duration of symptoms, a history of neck problems, and co-existing musculoskeletal disorders are poor prognostic factors for non-specific NP [7] and high baseline disability, heightened psychological stress, older age, radicular symptoms, litigation, and physically demanding work are prognostic for LBP [8]. Interestingly, prognostic factors across studies seem largely dependent on how outcomes were defined within the studies $[9,10]$. There is substantial variability amongst predictive models using different MCIDs on the Oswestry Disability Index (ODI) in the LBP population [10]. For NP, studies of prognostic factors have lacked predictors for outcome from a measure such as the Neck Disability Index (NDI) using different MCIDs [9].

We are unfamiliar with any modeling studies that have explored outcomes in patients who were seen via direct access to physical therapy. Direct access implies that patients are able to receive the services of a physical therapist without seeing a medical provider first. This may have importance, since evidence suggests that patients seen in a direct access environment may have unique characteristics [11], and since prognostic models may be reflective of this uniqueness. Consequently, the study objectives were to model prognostic factors that predict variations in the degree of recovery, defined by Trichotomized (divided into three equal parts, low, medium, and high) MCID categories, and insurance claims, defined by trichotomized cost categories (low, medium, and high), experienced by patients utilizing direct access physical therapy for spine pain. We also evaluated the impact on the predictive models when using higher and lower MCIDs, as reported by the literature for both the ODI and NDI. Results suggest modeling variations depend on different
\end{abstract} thresholds of success or claims costs.

\section{Materials and methods}

This study followed the Transparent Reporting of a multivariable prediction model for Individual Prognosis or Diagnosis (TRIPOD) initiative [12]. Key elements of the TRIPOD initiative include explanations of the source of data, participants, predictors, sample size, missing data, and statistical analysis methods.
This was a secondary database exploration of observational data that included patients with spine pain who chose direct access physical therapy. The sources of data were the ATI (Assessment Technologies Inc.) Patient Outcomes Registry paired with third party claims, where total claims paid were provided by an insurance payer.

Participants attended physical therapy via a direct access between January 2012 and December 2014. All patients received treatment across eight ATI physical therapy clinics within Greenville, South Carolina, USA. The database contained 603 patients with 447 who had unique total claims and patient outcomes data. Of these 447,63\% (280) received direct access physical therapy. The final sample size was 250 subjects. Thirty subjects were excluded because their initial ODI/NDI score was $\leqslant 10$; therefore, a 10 -point change was not possible for the MCID.

\subsection{Predictor variables}

Eleven prognostic factors were available for analysis. Many of these have been recognized as prognostic factors in the existing literature, including: age [13], gender [14-16], initial ODI or NDI score [10,17, 18], initial pain score [19], widespread pain [20-22], chronicity of symptoms $[14,18,20]$, the presence or absence of radicular pain [21,23], and whether patients used prescribed drugs [24]. Widespread pain was defined as the presence of strong leg pain, distal leg pain, or upper body pain [20] or a drawing of areas on a pain diagram [25]. Chronicity level described the duration of LBP $[14,18,20]$. We classified chronicity as acute ( $<30$ days), subacute (31-90 days), and chronic ( $>90$ days). These categories were similar to those of Bekkering et al. [14] who found that duration of symptoms was the most consistent factor across their prognostic modeling [14]. Radicular pain was defined as leg pain [21] or pain below the leg [23] in addition to back pain.

Because this was a direct access population, we included additional variables such as whether a participant saw a medical specialist, received imaging, or was managed with an active versus passive approach to physical therapy. Using a slight variation of an index measure created by Childs et al. [26], a participant's plan of care was considered active when greater than $75 \%$ of the interventions included active Current Procedural Terminology treatment codes. 


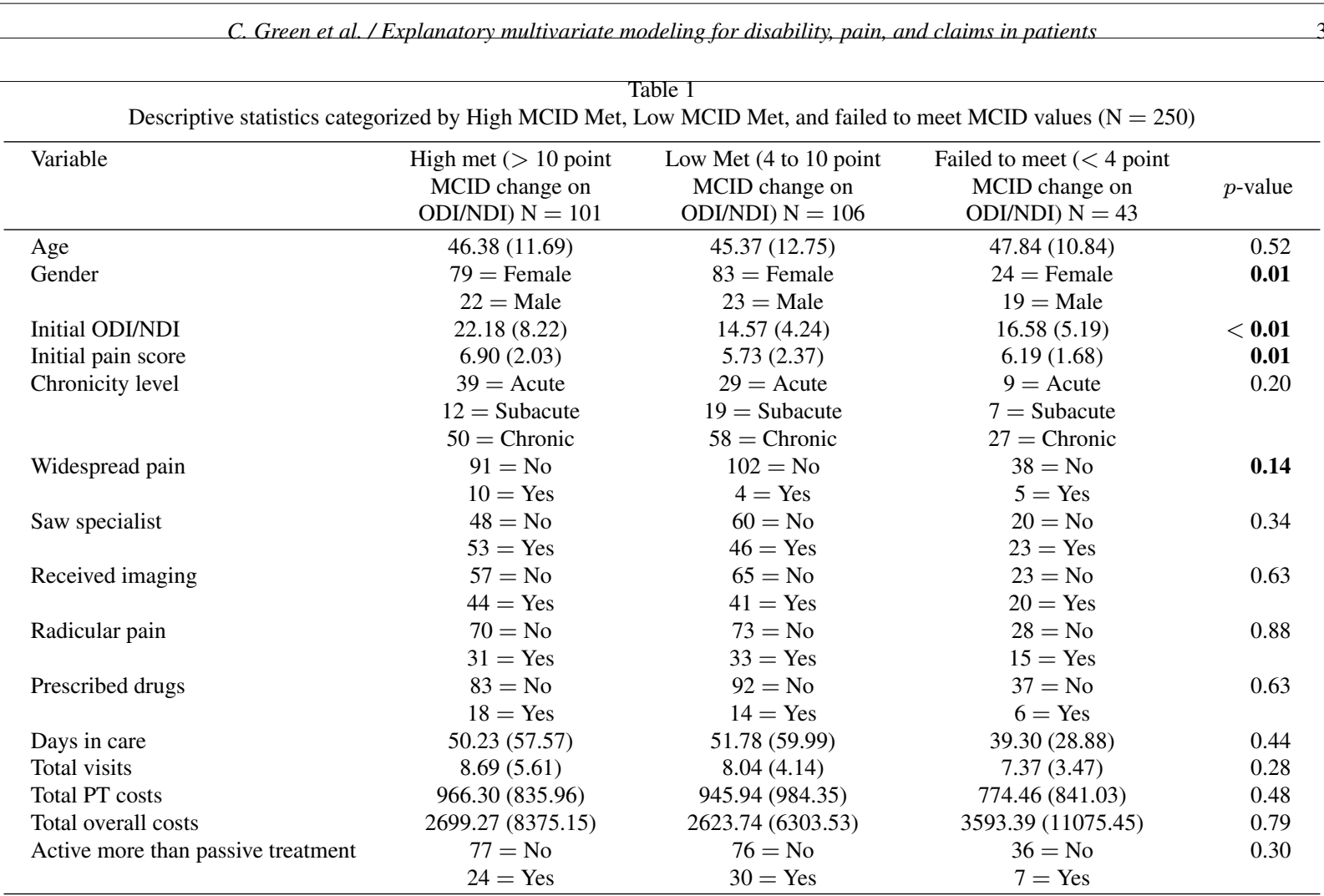

MCID - Minimally Clinically Important Difference; Bold indicates Significance $\leqslant 0.05$; acute (30 days), subacute (31 to 90 days), chronic ( $>$ 90 days).

\subsection{Outcome variables}

Two outcomes were: 1) change scores in disability, using ODI or NDI (hereby defined as ODI/NDI), and 2) insurance costs. ODI/NDI scores were recorded at baseline and discharge from physical therapy. ODI/ NDI raw scores ranged from 0 (no disability) to 50 (total disability).

ODI/NDI outcomes were categorized based on a range of reported MCIDs from the literature where improvement in the raw score typically included values in the range of 4 to 11 points [27-35]; with the most consistent value set at 10 points $[31,33,35,36]$. Based on these parameters from the literature, we operationally defined the ODI/NDI outcomes as: 1) High MCID Met ( $>10$ point change), Low MCID Met (4 to 10 point change), and Failed to Meet MCID ( $<4$ point change). Since no threshold data exists (to categorize levels of claims) in the literature, we trichotomized by tertiles (three equal representative groups by numbers) as low cost $(<\$ 793)$, moderate cost (\$1793-\$1881), and high cost $(>\$ 1881)$ to distinguish extreme differences.

\subsection{Missing values}

After refining the dataset to 250 , there were very few instances $(<1 \%)$ of missing data within the predictors and $0 \%$ missing data for outcomes variables. Missing data of the predictors were evaluated using Little's test for missing completely at random. Because there were so few instances of missing values we elected not to use imputation and instructed the statistical software to perform a complete case analysis, ignoring cases of missing values.

\subsection{Data analysis}

SPSS version 23.1 was used for all analyses. Descriptive statistics were used to describe all baseline sample characteristics with analysis of variance (ANOVA) and t-tests used to divide MCID categorical groups.

Univariate and multivariate multinomial logistic regression was used with the "failed to meet" MCID and lowest claims data tertiles used as the referent variables. Multinomial regression always uses a multiclass analysis when two distinct categorical variables are potential outcomes. Multinomial regression is used to explain the relationship between one nominal dependent variable and one or more independent variables. Prognostic variables that were statistically significant for 
Table 2

Bivariate relationships and multinomial logistic regression analyses: Odds ratios and $95 \%$ confidence intervals. Dependent Variable $=$ ODI/NDI Outcome per Utilization Trichotomized, Referent Category $=$ failed to meet MCID $(\mathrm{N}=250)$

\begin{tabular}{lccr}
\hline \multicolumn{1}{c}{ Variable (reference) } & $\begin{array}{c}\text { Multinomial categorization } \\
\text { outcome per utilization }\end{array}$ & $\begin{array}{c}\text { Odds ratio }(95 \% \\
\text { confidence unterval) }\end{array}$ & $p$-value \\
\hline Age & Higher MCID Met & $0.99(0.96,1.02)$ & 0.50 \\
Gender (Female) & Lower MCID Met & $0.98(0.95,1.01)$ & 0.26 \\
& Higher MCID Met & $2.84(1.32,6.11)$ & $<\mathbf{0 . 0 1}$ \\
Initial ODI/NDI score & Lower MCID Met & $2.86(1.34,6.10)$ & $<\mathbf{0 . 0 1}$ \\
Initial pain score & Higher MCID Met & $1.04(1.07,1.22)$ & $<\mathbf{0 . 0 1}$ \\
& Lower MCID Met & $0.91(0.84,0.98)$ & $\mathbf{0 . 0 1}$ \\
Widespread pain (No) & Higher MCID Met & $1.18(0.99,1.40)$ & 0.06 \\
& Lower MCID Met & $0.91(0.77,1.07)$ & 0.25 \\
Chronicity level & Higher MCID Met & $1.20(0.38,3.74)$ & 0.76 \\
(a = acute, b = subacute) & Lower MCID Met & $3.36(0.86,13.16)$ & 0.08 \\
& Higher MCID Met & a. $2.34(0.99,5.55)$ & 0.05 \\
Saw specialist (No) & & b. $0.93(0.33,2.63)$ & 0.89 \\
Received imaging (No) & Lower MCID Met & a. $1.50(0.63,3.60)$ & 0.36 \\
& b. $1.26(0.47,3.37)$ & 0.64 \\
Radicular pain (No) & Higher MCID Met & $1.04(0.51,2.13)$ & 0.91 \\
\multirow{2}{*}{ Prescribed drugs (No) } & Lower MCID Met & $1.50(0.74,3.06)$ & 0.26 \\
& Higher MCID Met & $1.13(0.56,2.31)$ & 0.75 \\
Active more than passive treatment (No) & Lower MCID Met & $1.38(0.68,2.82)$ & 0.38 \\
& Higher MCID Met & $1.21(0.57,2.58)$ & 0.62 \\
& Lower MCID Met & $1.19(0.56,2.51)$ & 0.66 \\
& Higher MCID Met & $0.75(0.28,2.04$ & 0.57 \\
& Lower MCID Met & $1.07(0.38,2.99)$ & 0.90 \\
& Higher MCID Met & $0.62(0.25,1.58)$ & 0.32 \\
& Lower MCID Met & $0.49(0.20,1.23)$ & 0.13 \\
\hline
\end{tabular}

MCID - Minimally Clinically Important Difference; Bold indicates Significance $\leqslant 0.05$; acute (30 days), subacute (31 to 90 days), chronic ( $>90$ days).

the univariate measures were carried forward to the multivariate analyses. For all analyses, a p value of less than 0.05 was used to discriminate statistical significance.

\section{Results}

Table 1 provides descriptive variables of the study for 250 patients with LBP and NP, categorized by MCID changes on the ODI/NDI, respectively. The groups were different $(p<0.05)$ based on gender and ODI/NDI score. Just over eighty-two percent (82.8\%) of the patients (206/250) were categorized in the High Met or Low Met MCID groups, while the remaining $17.2 \%(43 / 250)$ were categorized in the Failed to Meet MCID group.

Table 2 provides the bivariate, multinomial logistic regression analyses using ODI/NDI outcomes as the dependent variable while also trichotomizing High MCID Met and Low MCID Met. The Failed to Meet MCID was used as the referent category. Female gender and initial ODI/NDI score were found to be statistically significant in both the High and Low MCID
Met groups. Females had $2.84(95 \% \mathrm{CI}=1.32,6.11)$ greater odds of being in the High MCID and 2.86 (95\% $\mathrm{CI}=1.34,6.10)$ greater odds in being in the Low MCID Met. Individuals with a higher initial ODI/NDI score had $1.04(95 \% \mathrm{CI}=1.07,1.22)$ greater odds of being in a High MCID and lower odds of being in a Low MCID; 0.91 (95\% CI = 0.77, 1.07).

Table 3 represents the bivariate, multinomial logistic regression analyses using claims of care as the dependent variable while also trichotomizing High, Medium, and Low claims. The lowest trichotomized claims of care was used as the referent category $(<\$ 793)$. Not receiving imaging resulted in lower odds of being in the High and Medium claims groups respectively: 0.04 $(95 \% \mathrm{CI}=0.02,0.09)$ and $0.15(95 \% \mathrm{CI}=0.07$, 0.36 ). Other significant protective factors (against high claims) included not seeing a specialist 0.44 (95\% $\mathrm{CI}=0.24,0.82)$, not receiving prescribed drugs 0.36 $(95 \% \mathrm{CI}=0.14,0.18)$, and having a higher percentage of active versus passive treatment $0.38(95 \% \mathrm{CI}=$ $0.18,0.80$ ).

A hierarchical multivariate, multinomial logistic regression modeling was performed using ODI/NDI outcome per utilization trichotomized as the dependent 
Table 3

Bivariate relationships and multinomial logistic regression analyses: Odds ratios and 95\% confidence intervals. Dependent Variable $=$ Trichotomized Claims of Care, Referent Category $=$ Lowest Trichotomized Claims of Care $(\mathrm{N}=250)$

\begin{tabular}{|c|c|c|c|}
\hline Variable (reference) & $\begin{array}{l}\text { Multinomial categorization } \\
\text { outcome per utilization }\end{array}$ & $\begin{array}{c}\text { Odds ratio (95\% } \\
\text { confidence interval) }\end{array}$ & $p$-value \\
\hline \multirow[t]{2}{*}{ Age } & High claims & $0.99(0.96,1.01)$ & 0.29 \\
\hline & Medium claims & $0.99(0.97,1.02)$ & 0.56 \\
\hline \multirow[t]{2}{*}{ Gender (Female) } & High claims & $0.70(0.35,1.39)$ & 0.30 \\
\hline & Medium claims & $1.00(0.48,2.07)$ & 1.00 \\
\hline \multirow[t]{2}{*}{ Initial ODI/NDI score } & High claims & $1.01(0.97,1.06)$ & 0.54 \\
\hline & Medium claims & $1.01(0.96,1.05)$ & 0.80 \\
\hline \multirow[t]{2}{*}{ Initial pain score } & High claims & $1.00(0.87,1.15)$ & 0.96 \\
\hline & Medium claims & $1.03(0.90,1.19)$ & 0.66 \\
\hline \multirow[t]{2}{*}{ Widespread pain (No) } & High claims & $0.80(0.28,2.25)$ & 0.67 \\
\hline & Medium claims & $2.46(0.61,9.86)$ & 0.20 \\
\hline \multirow{4}{*}{$\begin{array}{l}\text { Chronicity level } \\
(\mathrm{a}=\text { acute }, \mathrm{b}=\text { subacute })\end{array}$} & High claims & a) $0.92(0.46,1.86)$ & 0.82 \\
\hline & & b) $0.92(0.39,2.19)$ & 0.85 \\
\hline & Medium claims & a) $1.59(0.80,3.16)$ & 0.19 \\
\hline & & b) $1.09(0.45,2.66)$ & 0.85 \\
\hline \multirow[t]{2}{*}{ Saw specialist (No) } & High claims & $0.44(0.24,0.82)$ & 0.01 \\
\hline & Medium claims & $0.95(0.51,1.77)$ & 0.87 \\
\hline \multirow[t]{2}{*}{ Received imaging (No) } & High claims & $0.04(0.02,0.09)$ & $<\mathbf{0 . 0 1}$ \\
\hline & Medium claims & $0.15(0.07,0.36)$ & $<\mathbf{0 . 0 1}$ \\
\hline \multirow[t]{2}{*}{ Radicular pain (No) } & High claims & $0.54(0.28,1.06)$ & 0.07 \\
\hline & Medium claims & $0.66(0.33,1.30)$ & 0.23 \\
\hline \multirow[t]{2}{*}{ Prescribed drugs (No) } & High claims & $0.36(0.14,0.18)$ & 0.02 \\
\hline & Medium claims & $0.88(0.32,2.40)$ & 0.80 \\
\hline \multirow{2}{*}{$\begin{array}{l}\text { Active more than } \\
\text { passive treatment (No) }\end{array}$} & High claims & $0.38(0.18,0.80)$ & 0.01 \\
\hline & Medium claims & $0.47(0.21,1.02)$ & 0.06 \\
\hline
\end{tabular}

Bold indicates Significance $\leqslant 0.05$; low claims $(<\$ 793)$, medium claims $(\$ 793-\$ 1881)$, high claims $(>\$ 1881)$.

variable, and "failed to meet" MCID as the referent category (Table 4). Among the High MCID Met group $(>10)$ as well as the Low MCID Met group (4-10): initial ODI/NDI score and female gender were found to be statistically significant. Higher initial ODI/NDI scores had $1.15(95 \% \mathrm{CI}=1.06,1.24)$ greater odds of being stratified within the High MCID Met group, and $0.91(95 \% \mathrm{CI}=0.84,0.99)$ greater odds of being in the Low MCID Met group. Females had 2.83 (95\% CI $=1.23,6.55)$ greater odds of being categorized within the High MCID Met group, as well as, 3.28 (95\% CI $=1.47,7.30)$ greater odds of being in the Low MCID Met group.

A hierarchical multinomial logistic regression modeling was performed using trichotomized claims of care as the dependent variable and the lowest trichotomized claims of care group $(<\$ 793)$ as the referent category (Table 5). Among the higher claims group ( $>$ \$1881) and the medium claims group (\$793-1881), not receiving imaging and a higher ratio of active treatment more than passive were both statistically significant and protective (less likely to have higher costs): $0.04(95 \% \mathrm{CI}=0.01,0.10)$ and $0.23(95 \% \mathrm{CI}=0.09$, 0.55) times lower odds of meeting the high claims
Table 4

Hierarchical Multinomial Logistic Regression Modeling including $p$ values, odds ratios, and $95 \%$ confidence intervals. Dependent Variable $=$ ODI/NDI Outcome per Utilization Trichotomized, Referent Category = failed to meet MCID $(\mathrm{N}=250)$

\begin{tabular}{lcr}
\hline \multicolumn{1}{c}{ Variable } & $\begin{array}{c}\text { Odds ratios }(95 \% \\
\text { confidence interval) }\end{array}$ & $p$-value \\
\hline Higher MCID Met $(>10)$ & & \\
Initial ODI/NDI score & $1.15(1.06,1.24)$ & $<\mathbf{0 . 0 1}$ \\
Initial pain score & $1.06(0.87,1.28)$ & 0.57 \\
Gender (Female) & $2.83(1.23,6.55)$ & $\mathbf{0 . 0 2}$ \\
Widespread pain (No) & $1.45(0.40,5.30)$ & 0.57 \\
Chronicity level & a. $2.35(0.92,5.99)$ & 0.08 \\
(a = acute, b $=$ subacute) & b. $0.78(0.26,2.40)$ & 0.67 \\
Active more than passive & $0.52(0.19,1.44)$ & 0.21 \\
treatment (Yes) & & \\
Lower MCID Met (4 to 10 points) & \\
Initial ODI/NDI score & $0.91(0.84,0.99)$ & $\mathbf{0 . 0 4}$ \\
Initial pain score & $0.93(0.78,1.11)$ & 0.42 \\
Gender (Female) & $3.28(1.47,7.30)$ & $<\mathbf{0 . 0 1}$ \\
Widespread pain (No) & $2.81(0.63,12.63)$ & 0.18 \\
Chronicity level & a. $1.25(0.50,3.16)$ & 0.63 \\
(a = acute, b = subacute) & b. $1.19(0.42,3.38)$ & 0.75 \\
Active more than passive & $0.49(0.19,1.28)$ & 0.14 \\
treatment (Yes) & & \\
\hline
\end{tabular}

MCID - Minimally Clinically Important Difference; Bold indicates Significance $\leqslant 0.05$; acute (30 days), subacute (31 to 90 days), chronic ( $>90$ days) 
Table 5

Hierarchical Multinomial Logistic Regression Modeling including $p$ values, odds ratios, and 95\% confidence intervals. Dependent Variable $=$ Trichotomized Claims of Care, Referent Category $=$ Lowest Trichotomized Claims of Care $(\mathrm{N}=250)$

\begin{tabular}{|llr}
\hline Variable & $\begin{array}{c}\text { Odds ratios }(95 \% \\
\text { confidence interval) }\end{array}$ & $p$-value \\
\hline High claims (>\$1881) & & \\
Radicular pain (No) & $0.55(0.25,1.23)$ & 0.15 \\
Received imaging (No) & $0.04(0.01,0.10)$ & $<\mathbf{0 . 0 1}$ \\
Saw specialist (No) & $0.63(0.30,1.32)$ & 0.22 \\
Prescribed drugs (No) & $0.81(0.27,2.43)$ & 0.71 \\
Active more than passive & $0.23(0.09,0.55)$ & $<\mathbf{0 . 0 1}$ \\
treatment (Yes) & & \\
Medium claims (\$793-1881) & & \\
Radicular pain (No) & $0.72(0.35,1.50)$ & 0.38 \\
Received imaging (No) & $0.13(0.05,0.31)$ & $<\mathbf{0 . 0 1}$ \\
Saw specialist (No) & $1.11(0.57,2.18)$ & 0.76 \\
Prescribed drugs (No) & $1.63(0.52,5.12)$ & 0.40 \\
Active more than passive & $0.39(0.17,0.89)$ & $\mathbf{0 . 0 3}$ \\
treatment (Yes) & & \\
Low claims (<\$793) & & \\
\hline Bold & &
\end{tabular}

Bold indicates Significance $\leqslant 0.05$.

group respectively as well as $0.13(95 \% \mathrm{CI}=0.05$, $0.31)$ and $0.39(95 \% \mathrm{CI}=0.17,0.89)$ lower odds of falling in the medium claims group respectively.

\section{Discussion}

We identified a variety of prognostic variables that were statistically significant for patients meeting high and Low MCID outcomes, which is a novel concept in that non-specific NP and LBP depended on the outcome selected. Our results indicate that the statistical significance of prognostic variables determined by different models depends largely on how outcome is defined, which corroborates with earlier work conducted by Schwind et al. [10] that concluded different MCID scores can affect the accuracy of prognostic factors when using the ODI as the outcome measure. In this study, the bivariate relationship and multinomial regression analyses revealed several statistically significant prognostic variables for the higher and the lower MCID met groups using the ODI/NDI as the dependent variable. Females had higher odds of meeting both the Low MCID and High MCID than males, which may suggest females could be predicted to have better outcomes. However, this finding runs counter to reports that the female gender is generally predicted to have poorer outcomes when experiencing LBP $[15,16,18]$. A possible explanation for this discrepancy is the present study included physical therapy intervention, whereas those studies did not include treatment by a physical therapist.
Our results suggest initial disability scores measured by the ODI/NDI can predict whether a patient will benefit from physical therapy treatment. Baseline ODI/NDI scores have been found as a prognostic factor elsewhere in the literature for both LBP and NP [10,17,37,38]. Cook et al. [17] included initial ODI score as a prognostic factor of interest to examine generic predictors of outcome in LBP patients. They found that lower baseline ODI scores were individual prognostic variables within two of 4 of their statistical models [17]. Schwind et al. [10] identified initial ODI score as a prognostic factor when using an MCID of 5 or 10 points on the ODI. Few studies have linked initial neck disability to outcome. According to De Pauw et al. [37], higher NDI scores at baseline are related to poorer outcomes. Likewise, patients with a NDI score of less than $18 / 50$ at baseline may be more likely to perceive improvement after treatment [38]. McLean et al. [7] concluded in their review of the literature that there is inconclusive evidence for the predictive power of baseline disability and NP for subjects with nonspecific NP, which is in agreement with the findings of Cecchi et al. [39].

The choice to trichotomize the MCID into High Met, Low Met, and Failed To Meet groups for the ODI/NDI was based on reported literature. A lower range of 4-6 points has been suggested to be a useful clinical cut-off score to determine if patients' disability had improved or not after a 6 week period [40]. This lower MCID threshold may be appropriate for patients with acute back pain because of its ability to establish a meaningful change after 6 weeks. Hägg et al. [33] found that an MCID of 10 was the lowest number they could identify within a $95 \%$ confidence interval. Ostelo and de Vet [34] proposed that acute sufferers of LBP may have higher ODI scores than those suffering from chronic LBP and suggested that 10 was an acceptable MCID value on the ODI, based on previous research. This recommendation was bolstered by the fact that Lauridsen et al. [32] found an average of 11 for their MCID across a stratification of patients with differing baseline ODI values and symptoms.

Five studies have reported on the MCID for the NDI [41-45] with scores ranging from 3.5 to 10 points depending on the study population. Pool et al. [43] found an MCID of 3.5 points by comparing NDI change scores and global perceived change using the area under the curve. Citing his expert opinion, the developer of the NDI, Vernon [46], concluded that 3.5 is the most appropriate MCID for the NDI. However, most studies report an MCID of 9.5 to 10 points in 
patients with mechanical NP or cervical radiculopa- 353 thy $[42,44,47]$. Because a range of values from 4 to 11 have been reported, it would stand to reason that a similar range of MCIDs for the ODI/NDI would be appropriate for use in our logistic regression analysis. Therefore, the MCIDs for the disability outcome measures were trichotomized as greater than 10 point change (High MCID Met), a 4 to10 point change (Low MCID Met), and $<4$ point change (Failed To Meet).

Patients in this study who did not have imaging had lower insurance claims. To our knowledge, imaging has not been used as a prognostic factor to determine claims. However, the suggestion that imaging is usually an unnecessary expense is supported elsewhere [48]. The most recent clinical practice guidelines (CPG) for LBP do not support imaging unless a patient is a surgical candidate [49].

An active approach to physical therapy versus a passive approach was associated with lower claims. An active approach to physical therapy for LBP has been suggested elsewhere as leading to better patient outcomes [50], which could explain why active approach is also less expensive. Linton et al. [51] demonstrated patients who received early active physical therapy were at a significantly reduced risk for developing chronic back pain. These findings have been supported by others in the literature $[52,53]$. In theory, a more efficient approach to therapy would require less visits and less money spent by both the patient and the insurance companies.

\subsection{Limitations}

Our results should be interpreted within the limitations of our study. There are other potential prognostic factors that were not accounted for in the present study. These include work status [20,22], psychosocial factors [16,54-58], and eligibility for the spinal manipulation clinical prediction rule (CPR) [17]. Additionally, other variables pertinent to LBP including pain, total visits, and self-perception of recovery were not used in this study. With multinomial regression, there is a chance some patients may have been misclassified. Finally, the retrospective nature of the data analysis did not allow blinding to the prognostic factors nor were predetermined cut points for disability or claims set beforehand.

\section{Conclusions}

Initial ODI/NDI scores and the female gender were prognostic for reaching both a low and high MCID met threshold in our secondary data analysis of patients seeking physical therapy via direct access. Additionally, an active versus passive approach to physical therapy and the absence of imaging were both associated with lower total claims of care. Future research should investigate the effect of prognostic factors that are consistently observed over various predictive models and their effect on claims in the direct access setting.

Key Points:

1. Our results suggest initial disability scores measured by the ODI/NDI can predict whether a patient will benefit from physical therapy treatment;

2. Not receiving imaging and a higher ratio of active treatment more than passive were associated with lower costs;

3. Variable MCID's did not change the predictors;

4. Medium and high costs groups had the same prognostic predictors

\section{Acknowledgments}

This work was supported by a MITRE Corporation FFRDC grant, coordinated through ATI, in South Carolina, USA.

\section{Conflict of interest}

None to report.

\section{References}

[1] Hoy D, March L, Woolf A, Blyth F, Brooks P, Smith E, Vos $\mathrm{T}$, Barendregt J, Blore J, Murray C, Burstein R, Buchbinder R. The global burden of neck pain: estimates from the Global Burden of Disease 2010 study. Ann Rheum Dis 2014; 73 1309-1315.

[2] Hoy D, March L, Brooks P, Blyth F, Woolf A, Bain C, Williams $G$. The global burden of low back pain: estimate from the Global Burden of Disease 2010 study. Ann Rheum Dis 2014; 73: 968-974.

[3] National Center for Health Statistics. Summary Health Statis tics for US. Adults: National Health Interview Survey, 2010 10, https//www.cdc.gov/nchs/data/series/sr_10/sr10_252.pdf.

[4] Côté P, Cassidy JD, Carroll LJ, Kristman V. The annua incidence and course of neck pain in the general population: A population-based cohort study. DOI: 10.1016/j.pain 2004.09.004.s

[5] Wasiak R, Pransky G, Verma S, Webster B. Recurrence of low back pain: definition-sensitivity analysis using administrative data. Spine (Phila Pa 1976) 2003; 28: 2283-2291.

[6] Stanton TR, Henschke N, Maher CG, Refshauge KM, Latimer $\mathrm{J}$, McAuley JH. After an episode of acute low back pain, recurrence is unpredictable and not as common as previously thought. Spine (Phila Pa 1976) 2008; 33: 2923-8. 
[7] McLean SM, May S, Moffett JK, Sharp DM, Gardiner E. Prognostic factors for progressive non-specific neck pain: a systematic review. Phys Ther Rev 2007; 12: 207-220.

[8] Hayden JA, Chou R, Hogg-Johnson S, Bombardier C. Systematic reviews of low back pain prognosis had variable methods and results-guidance for future prognosis reviews. J Clin Epidemiol 2009; 62: 781-796e1.

[9] Hill JC, Lewis M, Sim J, Hay EM, Dziedzic K. Predictors of poor outcome in patients with neck pain treated by physical therapy. Clin J Pain 2007; 23: 683-90.

[10] Schwind J, Learman K, O'Halloran B, Showalter C, Cook C. Different minimally important clinical difference (MCID) scores lead to different clinical prediction rules for the Oswestry disability index for the same sample of patients. J Man Manip Ther 2013; 21: 71-8.

[11] Ojha HA, Snyder RS, Davenport TE. Direct Access Compared With Referred Physical Therapy Episodes of Care: A Systematic Review. PHYS THER 2013; 94: 14-30.

[12] Moons KGM, Altman DG, Reitsma JB, Ioannidis JPA, Macaskill P, Steyerberg EW, Vickers AJ, Ransohoff DF, Collins GS. Transparent Reporting of a multivariable prediction model for Individual Prognosis Or Diagnosis (TRIPOD): Explanation and Elaboration. Ann Intern Med 2015; 162: W1

[13] Hayden JA, Dunn KM, van der Windt DA, Shaw WS. What is the prognosis of back pain? Best Pract Res Clin Rheumatol 2010; 24: 167-179.

[14] Bekkering GE, Hendriks HJM, van Tulder MW, Knol DL, Simmonds MJ, Oostendorp RAB, Bouter LM. Prognostic factors for low back pain in patients referred for physiotherapy: comparing outcomes and varying modeling techniques. Spine (Phila Pa 1976) 2005; 30: 1881-6.

[15] Lukas C, Dougados M, Combe B. Factors associated with a bad functional prognosis in early inflammatory back pain: results from the DESIR cohort. RMD Open 2016; 2: e000204.

[16] Campbell P, Foster NE, Thomas E, Dunn KM. Prognostic indicators of low back pain in primary care: Five-Year Prospective Study. J Pain 2013; 14: 873-883.

[17] Cook CE, Learman KE, O'Halloran BJ, Showalter CR, Kabbaz VJ, Goode AP, Wright AA. Which prognostic factors for low back pain are generic predictors of outcome across a range of recovery domains? Phys Ther 2013; 93: 32-40.

[18] Steenstra IA, Verbeek JH, Heymans MW, Bongers PM. Prognostic factors for duration of sick leave in patients sick listed with acute low back pain: a systematic review of the literature. Occup Environ Med 2005; 62: 851-60.

[19] Melloh M, Elfering A, Egli Presland C, Roeder C, Barz T, Rolli Salathé C, Tamcan O, Mueller U, Theis JC. Identification of prognostic factors for chronicity in patients with low back pain: a review of screening instruments. Int Orthop 2009; 33: 301-13.

[20] Dunn KM, Jordan KP, Croft PR. Contributions of prognostic factors for poor outcome in primary care low back pain patients. Eur J Pain 2011; 15: 313-319.

[21] George S, Bialosky J, Donald D. The centralization phenomenon and fear-avoidance beliefs as prognostic factors for acute low back pain. J Orthop Sports Phys Ther 2005; 35 : 844-845; author reply 845-847.

[22] Grotle M, Foster NE, Dunn KM, Croft P. Are prognostic indicators for poor outcome different for acute and chronic low back pain consulters in primary care? Pain 2010; 151: 790797.

[23] Shaw WS, Pransky G, Fitzgerald TE. Early prognosis for low back disability: intervention strategies for health care providers. Disabil Rehabil 2001; 23: 815-28.
[24] Traeger AC, Henschke N, Hübscher M, Williams CM, Kam per SJ, Maher CG, Moseley GL, McAuley JH. Estimating the Risk of Chronic Pain: Development and Validation of a Prognostic Model (PICKUP) for Patients with Acute Low Back Pain. PLOS Med 2016; 13: e1002019.

[25] Haxby Abbott J, Kingan E-M. Accuracy of physical thera pists' prognosis of low back pain from the clinical examination: a prospective cohort study. J Man Manip Ther 2014; 22 154-61.

[26] Childs JD, Fritz JM, Wu SS, Flynn TW, Wainner RS, Robert son EK, Kim FS, George SZ. Implications of early and guideline adherent physical therapy for low back pain on utilization and costs. BMC Health Serv Res 2015; 15: 150.

[27] Beurskens AJ, de Vet HC, Köke AJ. Responsiveness of functional status in low back pain: a comparison of different in struments. Pain 1996; 65: 71-6.

[28] Maughan EF, Lewis JS. Outcome measures in chronic low back pain. Eur Spine J 2010; 19: 1484-1494.

[29] Parker SL, Adogwa O, Mendenhall SK, Shau DN, Anderson WN, Cheng JS, Devin CJ, McGirt MJ. Determination of min imum clinically important difference (MCID) in pain, disabil ity, and quality of life after revision fusion for symptomatic pseudoarthrosis. Spine J 2012; 12: 1122-1128.

[30] Resnik L, Dobrzykowski E. Guide to outcomes measurement for patients with low back pain syndromes. J Orthop Sports Phys Ther 2003; 33: 307-316; discussion 317-318.

[31] Eleswarapu A, Divi S, Dirschl D, Mok J. How Effective is Physical Therapy for Common Low Back Pain Diagnoses? A Multivariate Analysis of 4597 Patients. Spine (Phila Pa 1976) 2016; 41: 1325-1329.

[32] Lauridsen HH, Hartvigsen J, Manniche C, Korsholm L Grunnet-Nilsson N. Responsiveness and minimal clinically important difference for pain and disability instruments in low back pain patients. BMC Musculoskelet Disord 2006; 7: 82 .

[33] Hägg O, Fritzell P, Nordwall A. The clinical importance of changes in outcome scores after treatment for chronic low back pain. Eur Spine J 2003; 12: 12-20.

[34] Ostelo RWJG, de Vet HCW. Clinically important outcomes in low back pain. Best Pract Res Clin Rheumatol 2005; 19 593-607.

[35] Khan M, Bashir M, Kumar R, Enam S. Enduring improvement in Oswestry Disability Index outcomes following lum bar microscopic interlaminar decompression: An appraisa of prospectively collected patient outcomes. J Cranioverteb Junction Spine 2015; 6: 195.

[36] Ostelo RWJG, Deyo RA, Stratford P, Waddell G, Croft P, Von Korff M, Bouter LM, De Vet HC. Interpreting Change Scores for Pain and Functional Status in Low Back Pain. Spine (Phila Pa 1976) 2008; 33: 90-94.

[37] De Pauw R, Kregel J, De Blaiser C, Van Akeleyen J, Logghe $\mathrm{T}$, Danneels L, Cagnie B. Identifying prognostic factors predicting outcome in patients with chronic neck pain after multimodal treatment: A retrospective study. Man Ther 2015; 20 592-597.

[38] Hanney WJ, Kolber MJ, George SZ, Young I, Patel CK, Cleland JA. Development of a preliminary clinical prediction rule to identify patients with neck pain that may benefit from a standardized program of stretching and muscle performance exercise: a prospective cohort study. Int J Sports Phys The 2013; 8: 756-76.

[39] Cecchi F, Molino-Lova R, Paperini A, Boni R, Castagnoli C, Gentile J, Pasquini G, Macchi C. Predictors of short- and long-term outcome in patients with chronic non-specific neck pain undergoing an exercise-based rehabilitation program: a 
prospective cohort study with 1-year follow-up. Intern Emerg 56 Med 2011; 6: 413-21.

[40] Beurskens AJHM, de Vet HCW, Köke AJA. Responsiveness of functional status in low back pain: A comparison of different instruments. Pain 1996; 65: 71-76.

[41] Stratford P, Riddle D, Binkley J, Spadoni G, Westaway MDPB. Using the Neck Disability Index to make decisions concerning individual patients. Physiother Canada.

[42] Cleland JA, Childs JD, Whitman JM. Psychometric Properties of the Neck Disability Index and Numeric Pain Rating Scale in Patients With Mechanical Neck Pain. Arch Phys Med Rehabil 2008; 89: 69-74.

[43] Pool JJM, Ostelo RWJG, Hoving JL, Bouter LM, de Vet HCW. Minimal clinically important change of the Neck Disability Index and the Numerical Rating Scale for patients with neck pain. Spine (Phila Pa 1976) 2007; 32: 3047-51.

[44] Young BA, Walker MJ, Strunce JB, Boyles RE, Whitman JM, Childs JD. Responsiveness of the Neck Disability Index in patients with mechanical neck disorders. Spine J 2009; 9: 802808.

[45] Cleland JA, Fritz JM, Whitman JM, Palmer JA. The reliability and construct validity of the Neck Disability Index and patient specific functional scale in patients with cervical radiculopathy. Spine (Phila Pa 1976) 2006; 31: 598-602.

[46] Vernon H. The Neck Disability Index: State-of-the-Art, 19912008. J Manipulative Physiol Ther 2008; 31: 491-502.

[47] Childs JD, Cleland JA. Development and application of clinical prediction rules to improve decision making in physical therapist practice. Phys Ther 2006; 86: 122-31.

[48] Kendrick D, Bentley E, Kerslake R, Miller P, Pringle M. Radiography of the lumbar spine in primary care patients with low back pain: randomised controlled trial. Bmj 2001; 322: 400-405.

[49] Chou R, Qaseem A, Snow V, Casey D, Shekelle JTP, Owens DK. Clinical Guidelines Diagnosis and Treatment of Low Back Pain: A Joint Clinical Practice Guideline from the American College of Physicians and the American.
[50] Bekkering GE, Engers AJ, Wensing M, Hendriks HJ, van Tul der MW, Oostendorp RA, Bouter LM. Development of an implementation strategy for physiotherapy guidelines on low back pain. Aust J Physiother 2003; 49: 208-214.

[51] Linton SJ, Hellsing A-L, Andersson D. A controlled study of the effects of an early intervention on acute musculoskeleta pain problems. Pain 1993; 54: 353-359.

[52] Wand BM, Bird C, McAuley JH, Doré CJ, MacDowell M, De Souza LH. Early intervention for the management of acute low back pain: a single-blind randomized controlled tria of biopsychosocial education, manual therapy, and exercise. Spine (Phila Pa 1976) 2004; 29: 2350-6.

[53] Hagen EM, Eriksen HR, Ursin H. Does early intervention with a light mobilization program reduce long-term sick leave for low back pain? Spine (Phila Pa 1976) 2000; 25: 1973-6.

[54] Burton AK, McClune TD, Clarke RD, Main CJ. Long-term follow-up of patients with low back pain attending for ma nipulative care: Outcomes and predictors. Man Ther 2004; 9 : 30-35.

[55] Foster NE, Bishop A, Thomas E, Main C, Horne R, Wein man J, Hay E. Illness perceptions of low back pain patients in primary care: what are they, do they change and are they associated with outcome? Pain 2008; 136: 177-87.

[56] Schultz IZ, Crook J, Meloche GR, Berkowitz J, Milner R, Zuberbier OA, Meloche W. Psychosocial factors predictive of occupational low back disability: Towards development of a return-to-work model. Pain 2004; 107: 77-85.

[57] Henschke N, Maher CG, Refshauge KM, Herbert RD, Cum ming RG, Bleasel J, York J, Das A, McAuley JH. Prognosis in patients with recent onset low back pain in Australian primary care: inception cohort study. BMJ 2008; 337: a171.

[58] Ramond A, Bouton C, Richard I, Roquelaure Y, Baufreton C, Legrand E, Huez JF. Psychosocial risk factors for chronic low back pain in primary care-a systematic review. Fam Pract 2011; 28: 12-21. 